10,09

\title{
Ab initio и экспериментальное исследование электронных, оптических и колебательных свойств $\mathrm{CdGa}_{2} \mathrm{Te}_{4}$
}

\author{
(C) 3.А. Джахангирли ${ }^{1,2}$, Т.Г. Керимова ${ }^{1}$, И.А. Мамедова ${ }^{1}$, С.А. Набиева ${ }^{1,3}$, Н.А. Абдуллаев ${ }^{1,4}$ \\ ${ }^{1}$ Институт ффизики НАН Азербайджана, \\ Баку, Азербайджан \\ ${ }^{2}$ Азербайджанский технический университет, \\ Баку, Азербайджан \\ ${ }^{3}$ Академия министерства по чрезвычайным ситуациям Азербайджанской Республики, \\ Баку, Азербайджан \\ ${ }^{4}$ Бакинский государственный университет, \\ Баку, Азербайджан \\ E-mail: zakircahangirli@yahoo.com
}

Поступила в Редакцию 6 февраля 2020 г.

В окончательной редакции 6 февраля 2020 г.

Принята к публикации 6 апреля 2020 г.

\begin{abstract}
Электронные, оптические и решеточные колебательные свойства $\mathrm{CdGa}_{2} \mathrm{Te}_{4}$ исследованы экспериментально с использованием спектральной эллипсометрии, комбинационного рассеяния (КР) и инфракрасной (ИК) спектроскопии, а также теоретически с использованием теории функционала плотности (DFT). Семь раман-активных мод и одиннадцать ИК-активных мод были обнаружены и идентифицированы из рассмотрения точечной группы симметрии. На основе анализа электронного спектра и плотности энергетических состояний определен характер химической связи в этом полупроводниковом соединении. Теоретически рассчитанные результаты сравнены с экспериментальными данными настоящей работы и с результатами имеющихся в литературе экспериментальными данными, полученными методами инфракрасной спектроскопии и комбинационного рассеяния света.
\end{abstract}

Ключевые слова: $\mathrm{CdGa}_{2} \mathrm{Te}_{4}$, раман, ИК-активные моды, дисперсия фононов, плотность состояний.

DOI: $10.21883 /$ FTT.2020.08.49613.024

\section{1. Введение}

Тройные соединения $\mathrm{A}^{\mathrm{II}} \mathrm{B}_{2}^{\mathrm{III}} \mathrm{C}_{4}^{\mathrm{VI}}$ привлекают внимание исследователей в связи их перспективностью применения в электрооптических, оптоэлектронных и нелинейных оптических приборах [1].

Для этих соединений характерны оптическая анизотропия, двулучепреломление, значительные величины коэффициентов нелинейной восприимчивости, высокая фоточувствительность, яркая люминесценция. Наличие указанных свойств в сочетании с значительными величинами ширины запрещенной зоны выдвигают эти соединения в ряд перспективных материалов для использования в полупроводниковом приборостроении [1]. Поэтому, исследования физических свойств этих соединений является важной задачей. Особое место занимают исследования электронных и фононных состояний как экспериментально, так и теоретически. Знание фононных спектров, их зависимость от температуры, давления и других внешних факторов важны при объяснении механизмов теплоемкости, теплового расширения, теплопроводности, поглощения звука и др. Совместные теоретические и экспериментальные исследования фононных спектров, динамики решетки позволяют получить информацию о фононных частотах, фонон-электронном, фонон-фононном взаимодействиях и др.
Кристаллы $\mathrm{CdGa}_{2} \mathrm{Te}_{4}$ являются одним из представителей класса соединений. Оптические свойства и электронная зонная структура $\mathrm{CdGa}_{2} \mathrm{Te}_{4}$ изучены $a b$ initio в [2]. В результате расчетов оказалось, что $\mathrm{CdGa}_{2} \mathrm{Te}_{4}$ имеет прямую запрещенную зону шириной $1.047 \mathrm{eV}$, где потолок валентной зоны и дно зоны проводимости локализованы в точке $\Gamma$ зоны Бриллюэна, а экспериментальное определенное значение равно $1.445 \mathrm{eV}$ $(T=11 \mathrm{~K})$. Из данных расчетов также следует, что верхняя часть валентной зоны формируется гибридизированными $p$-состояниями $\mathrm{Ga}$ и Те, а дно зоны проводимости формируется $s$-состояниями $\mathrm{Ga}$, состояниями Те и с малым смешиванием $s$-состояний $\mathrm{Cd}$. Из анализа оптических свойств в интервале $2-20 \mathrm{eV}$ авторы показали, что $\mathrm{CdGa}_{2} \mathrm{Te}_{4}$ в промежуточной области энергий проявляет себя как анизотропный материал. Оптические свойства также исследованы в [3] методами оптического поглощения, спектроскопической эллипсометрии и электроотражения. Спектр комплексной диэлектрической функции измерен методом спектральной эллипсометрии в области энергий от 1.2 до $5.2 \mathrm{eV}$. Также рассчитана электронная зонная структура с использованием эмпирического псевдопотенциала: валентная зона разделена на две подзоны, которые отдалены друг от друга на $7 \mathrm{eV}$. Определено расщепление кристаллического поля $0.2 \mathrm{eV}$, а спин-орбитальное расщепление составляет $0.9 \mathrm{eV}$. Оптические и люми- 


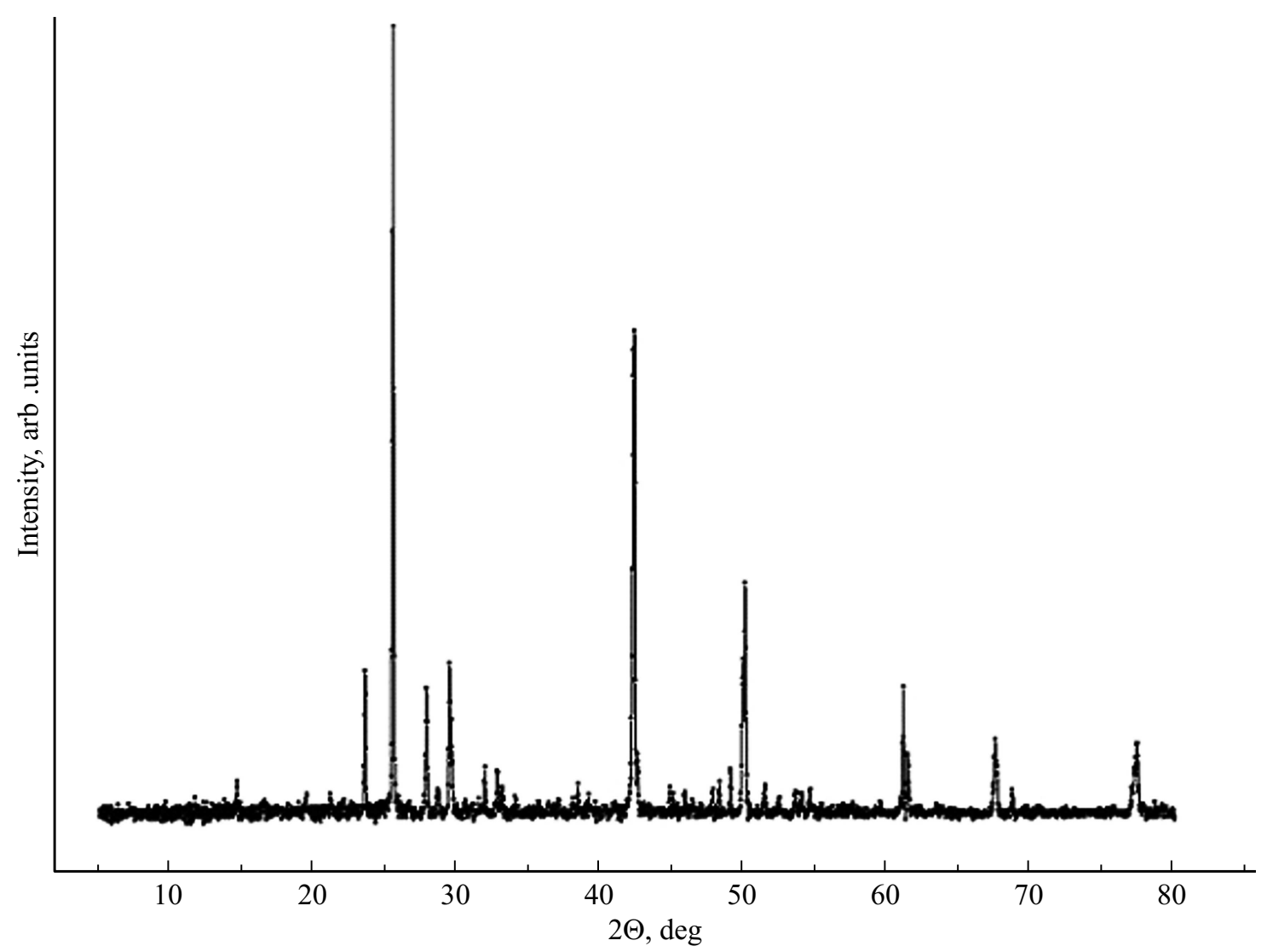

Рис. 1. Рентгенограмма $\mathrm{CdGa}_{2} \mathrm{Te}_{4}$.

несцентные свойства изучены также в [4]. Ширина запрещенной зоны при комнатной температуре равна $1.35 \mathrm{eV}$. Из люминесцентных измерений определено положение глубоких донорных состояний ниже дна зоны проводимости $(\sim 190 \mathrm{meV})$ и акцепторные уровни выше валентной зоны (50 и $86 \mathrm{meV})$. В работе [5] для ширины запрещенной зоны получено значение $1.25 \mathrm{eV}$. Из расчетов парциальной плотности состояний сделан вывод о главной роли $p-d$-гибридизации в формировании ширины запрещенной зоны. Диэлектрические постоянные рассчитаны как $\varepsilon_{1}(0)_{\perp}=10.94, \varepsilon_{1}(0)_{\|}=7.71$, коэффициенты преломления $n(0)_{\perp}=3.3$ и $n(0)_{\|}=2.77$. $\mathrm{B}$ [6] для ширины запрещенной зоны получено значение $1.5 \mathrm{eV}$, а в [7] из расчетов получено значение $1.9 \mathrm{eV}$. Как видно, имеется разброс в значениях оптических параметров (ширины запрещенной зоны и т.д.). Исследования ИК-отражения в $\mathrm{CdGa}_{2} \mathrm{Te}_{4}$ проведены в поляризованном излучении в [8]. Обнаружены десять мод: 5 мод для поляризации $E \| c, 5$ мод для поляризации $E \perp c$.

Ранее нами были рассчитаны дисперсия фононов и плотность фононных состояний $\mathrm{CdGa}_{2} \mathrm{Se}_{4}$ [9] и $\mathrm{CdGa}_{2} \mathrm{~S}_{4}[10]$. В настоящей работе представлены результаты $a b$ initio расчета плотности фононных состояний и дисперсии фононов в высокосимметричных точках и линиях зоны Бриллюэна (ЗБ) $\mathrm{CdGa}_{2} \mathrm{Te}_{4}$ с целью выяснения влияния анионов $(\mathrm{Se}, \mathrm{S}, \mathrm{Te})$ на динамические свойства. Как нам известно, в литературе отсутствует $a b$ initio расчет фононного спектра $\mathrm{CdGa}_{2} \mathrm{Te}_{4}$.

Несмотря на большой объем экспериментальных данных по кристаллам $\mathrm{CdGa}_{2} \mathrm{Te}_{4}$, в литературе отсутствуют систематические теоретические исследования, связанные с расчетами их электронно-энергетической структуры и описаниями основных особенностей химической связи в них, а также динамических характеристик, определяющие термодинамические характеристики и оптические свойства кристаллов в инфракрасной области. В данной статье представлены результаты экспериментального и теоретического исследования электронных структур, оптических и динамических свойств кристалла $\mathrm{CdGa}_{2} \mathrm{Te}_{4}$.

\section{2. Методика эксперимента, кристаллическая структура и метод расчета}

Образцы для измерений были синтезированы из исходных компонентов $\mathrm{Cd}, \mathrm{Ga}$ и Те взятых в стехиометрическом соотношении в графитизированных кварцевых ампулах. Образцы имели форму пластинок с размерами $5 \times 5 \mathrm{~mm}$. Рентгендифрактометрические исследования проводились на приборе Bruker D8 Advance. $\mathrm{CdGa}_{2} \mathrm{Te}_{4}$ кристаллизуется в тетрагональной структуре (пр. гр.I 4). 
Таблица 1. Структурные параметры $\mathrm{CdGa}_{2} \mathrm{Te}_{4}$

\begin{tabular}{l|l|l|c}
\hline$a, \AA$ & $c, \AA$ & $c / a$ & Литература \\
\hline 6.10 & 11.70 & 1.918 & {$[11]$} \\
6.18 & 12.22 & 1.977 & {$[5]$} \\
6.278 & 12.00 & 1.911 & {$[2]$} \\
6.04 & 12.135 & 2.005 & Данная работа (эксп.) \\
6.12 & 11.86 & 1.94 & Данная работа (теория)
\end{tabular}

На рис. 1 представлена рентгенограмма $\mathrm{CdGa}_{2} \mathrm{Te}_{4}$. Полученные нами параметры решетки (табл. 1) согласуются с результатами авторов $[2,5,11]$. Измерения спектров комбинационного рассеяния в $\mathrm{CdGa}_{2} \mathrm{Te}_{4}$ были проведены на конфокальном рамановском микроспектрометре Nanofinder 30 (Tokyo Instr., Japan). В качестве источника возбуждения использовался лазер $\mathrm{Nd}$ :YAG с выходной длиной волны излучения $\lambda=532 \mathrm{~nm}$ и максимальной мощностью $10 \mathrm{~mW}$. Спектральное разрешение было не хуже $0.5 \mathrm{~cm}^{-1}$. Детектором рассеянного излучения являлась охлаждаемая (термоэлектрическим способом до $\left.-100^{\circ} \mathrm{C}\right) \mathrm{CCD}$ камера (1024 на 12 pixel), работающая в режиме счета фотонов. Измерения спектров комбинационного рассеяния света производились в геометрии обратного рассеяния. Спектры ИК-отражения были сняты на приборе ИК-спектрофотометре ThermoNicolet (с приставкой micro-specular reflectance accessory -2x 186-0324). Спектральное разрешение $4 \mathrm{~cm}^{-1}$, угол падения $\alpha=20^{\circ}$, количество сканов 256, приемник DTGS (полиэтиленовое окно). В процессе эксперимента для удаления паров воды использовался генератор сухого воздуха: Parker Balston — 74-5041. Ab initio расчеты электронных и оптических свойств проводились на основе DFT, а динамические характеристики с помощью теории возмущений функционала плотности DFPT (Density Functional PerturbationTheory) [12-14] c использованием метода псевдопотенциала на основе плоских волн, реализованного в коде ABINIT [15]. В этой работе были использованы сохраняющие нормы псевдопотенциалы Hartwigsen-Goedecker-Hutter [16]. Обменно-корреляционное взаимодействие описывалось в обобщенном градиентном приближении (GGA) [17]. В разложении волновых функций были учтены плоские волны с энергией до $80 \mathrm{Ry,} \mathrm{что} \mathrm{обеспечивает} \mathrm{хорошую}$ сходимость полной энергии. Суммирования по ЗБ выполнялись на сетке $4 \times 4 \times 4$ Монкхорст-Пака [18].

Параметры решетки и равновесные положения атомов в элементарной ячейке определялись минимизацией сил Геллмана-Фейнмана. Равновесные положения атомов в элементарной ячейке были найдены методом BFGS (Broyden-Fletcher-Goldfarb-Shanno) с использованием экспериментальных данных в качестве начальных значений. Процесс минимизации продолжался до тех пор, пока силовые модули не оказывались меньше $10^{-8} \mathrm{Ry} /$ Bohr. При расчете плотности фононных состояний была использована сетка $40 \times 40 \times 40$ точек в ЗБ. Для получения LO-TO-расщепления в центре ЗБ при полярных модах, учтено дальнодействующее кулоновское поле и в динамическую матрицу добавлен неаналитический член, который зависит от тензоров эффективного заряда Борна и электронной диэлектрической проницаемости. Зависимость сходимости полной энергии и сил Геллман-Фейнмана от сетки Монхорста-Пака и от максимальной энергии плоских волн с учетом оптимальной употребляемой машинной времени для вычислений показали, что сетка $4 \times 4 \times 4$ и максимальная энергия плоских волн $80 \mathrm{Ry}$ в разложении волновых функций дают достаточно хорошие результаты для электронных и динамических свойств $\mathrm{CdGa}_{2} \mathrm{Te}_{4}$.

\section{3. Электронная структура и оптические функции}

Зонная структура и проектированные на атомы парциальные плотности состояний (PDOS) показаны на рис. 2 и 3 соответственно. Зонная структура $\mathrm{CdGa}_{2} \mathrm{Te}_{4}$ обладают рядом характерных особенностей: валентная зона состоит из четырех подзон. Нижняя подзона шириной $\sim 1 \mathrm{eV}$, состоящая из четырех зон, отдалена от остальных энергетическим зазором $\sim 3 \mathrm{eV}$ и происходит из $s$-состояний халькогена Те с небольшим вкладом $d$-состояний атома $\mathrm{Cd}$. Как следует из PDOS бездисперсионные состояния около $-8 \mathrm{eV}$ обязаны своим происхождением $d$-состояний атомов $\mathrm{Cd}$. Анализ парциальной плотности состояний показывает, что группа валентных

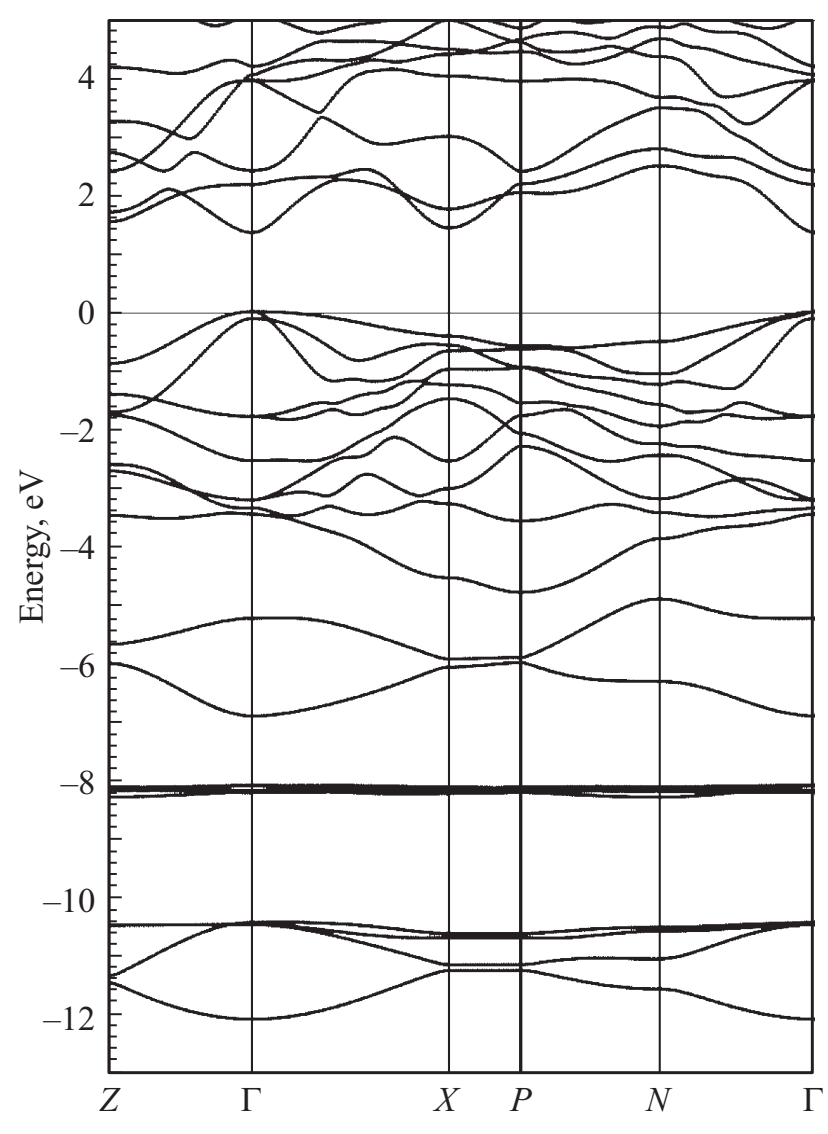

Рис. 2. Зонная структура $\mathrm{CdGa}_{2} \mathrm{Te}_{4}$. 

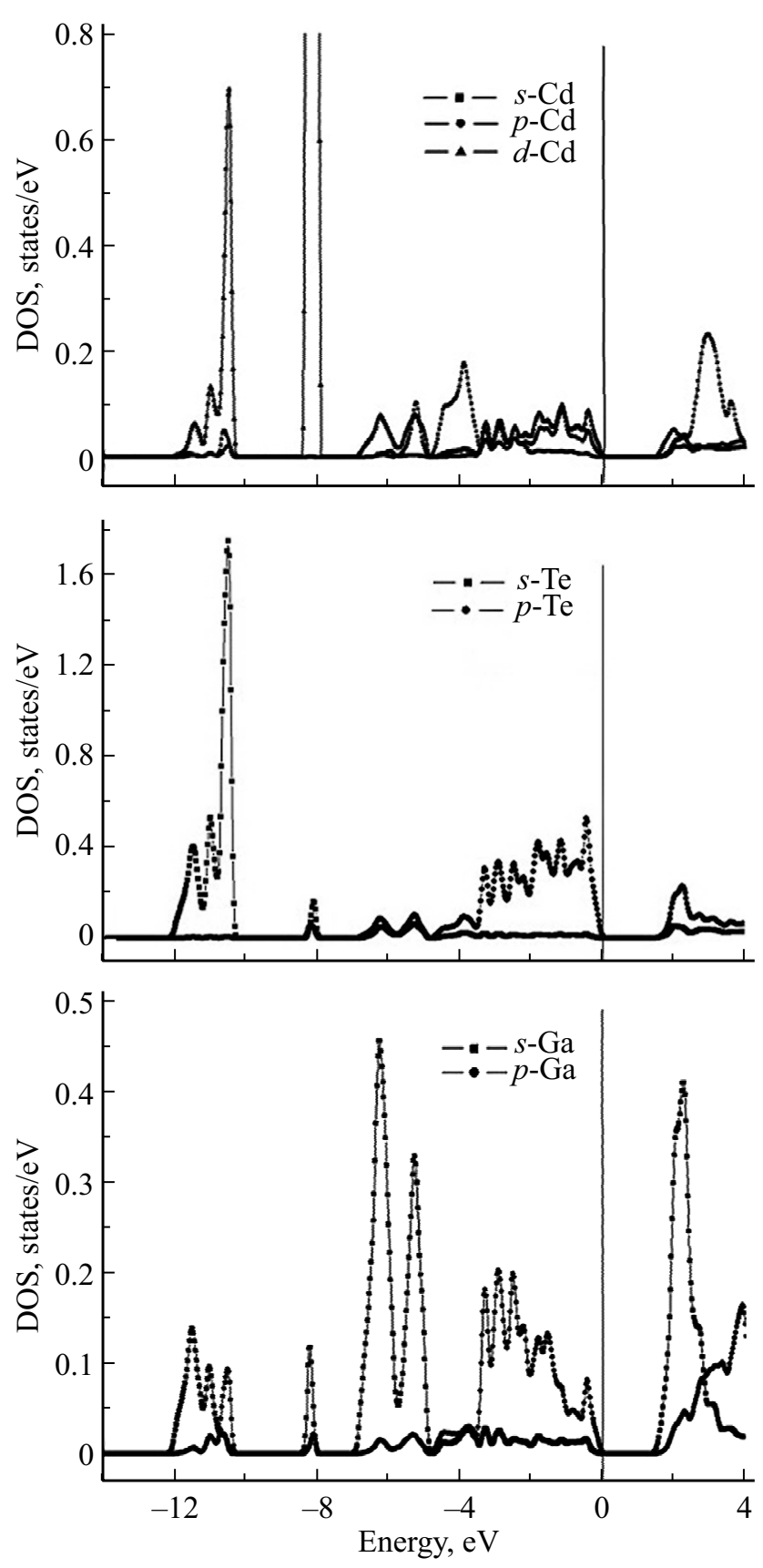

Рис. 3. Парциальные плотности состояний атомов $\mathrm{Cd}, \mathrm{Ga}$ и Те. Уровень Ферми соответствует нулевой энергии.

зон около $-6 \mathrm{eV}$ происходит из $s$-состояний $\mathrm{Ga}$. Наконец, самую верхнюю группу валентных зон можно приписать $p$-состояниям атома Те с малым вкладом $p$ и $d$-состояний $\mathrm{Cd}$ и $p$-состояний $\mathrm{Ga}$. Потолок валентной зоны и абсолютный минимум зоны проводимости находится в точке $Г$ и соответственно, $\mathrm{CdGa}_{2} \mathrm{Te}_{4}$ является прямозонным полупроводником с шириной запрещенной зоны $\sim 1.5 \mathrm{eV}$. Из парциальной плотности состояний также видно, что потолок валентной зоны в основном состоит из $p$-состояний атома Те, а дно зоны проводимости состоит из $s$-состояний атома $\mathrm{Ga}$ с некоторой примесью $p$-состояний атома Те. Из него же следует, что p-состояния атома $\mathrm{Ga}$ гибридизуется с p-состояниями атома Те.

Сравнение рассчитанных оптических свойств с экспериментами позволяет нам проверить правильность нашей зонной структуры. Собственные значения и собственные векторы полученные из расчетов зонной структуры использовались для определения действительной и мнимой частей диэлектрической функции, коэффициента поглощения и показателя преломления. Рассчитанные и измеренные оптические функции $\mathrm{CdGa}_{2} \mathrm{Te}_{4}$ показаны на рис. 4, 5 и 6. Отметим, что только прямые переходы, которые сохраняют импульс, включены в наши расчеты для оптических свойств. Непрямые переходы с участием фононов оказывают очень слабое влияние на край поглощения, так как $\mathrm{CdGa}_{2} \mathrm{Te}_{4}$ является прямозонным полупроводником.

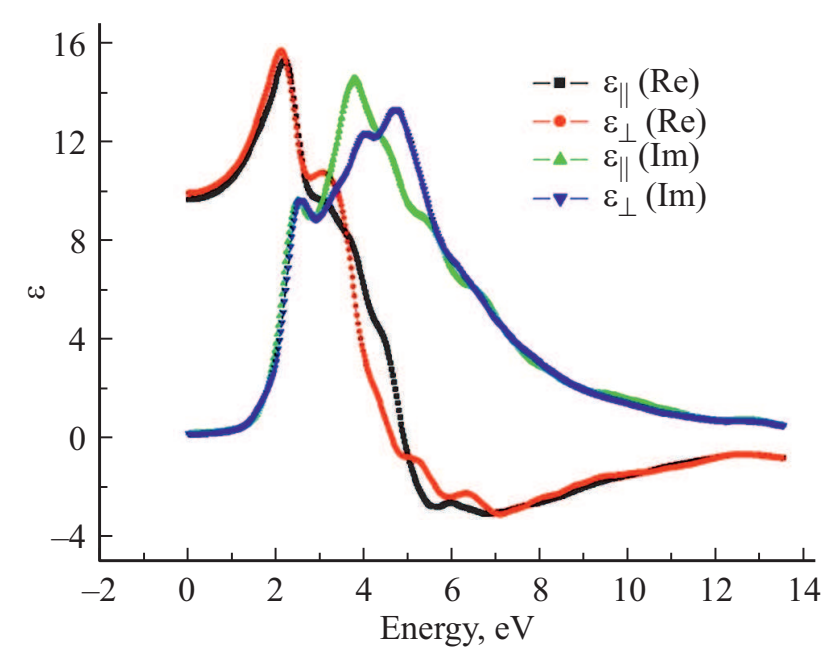

Рис. 4. Реальные и мнимые части диэлектрической функции $\mathrm{CdGa}_{2} \mathrm{Te}_{4}(\|-$ вдоль тетрагональной оси $c, \perp-$ перпендикулярно тетрагональной оси $c$ ).

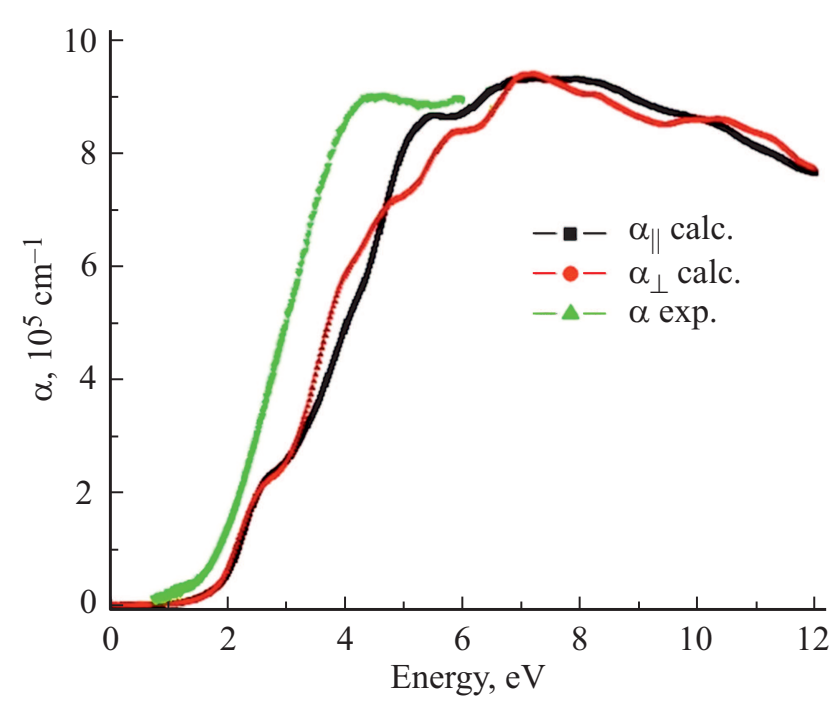

Рис. 5. Спектр оптического поглощения $\mathrm{CdGa}_{2} \mathrm{Te}_{4}$. 

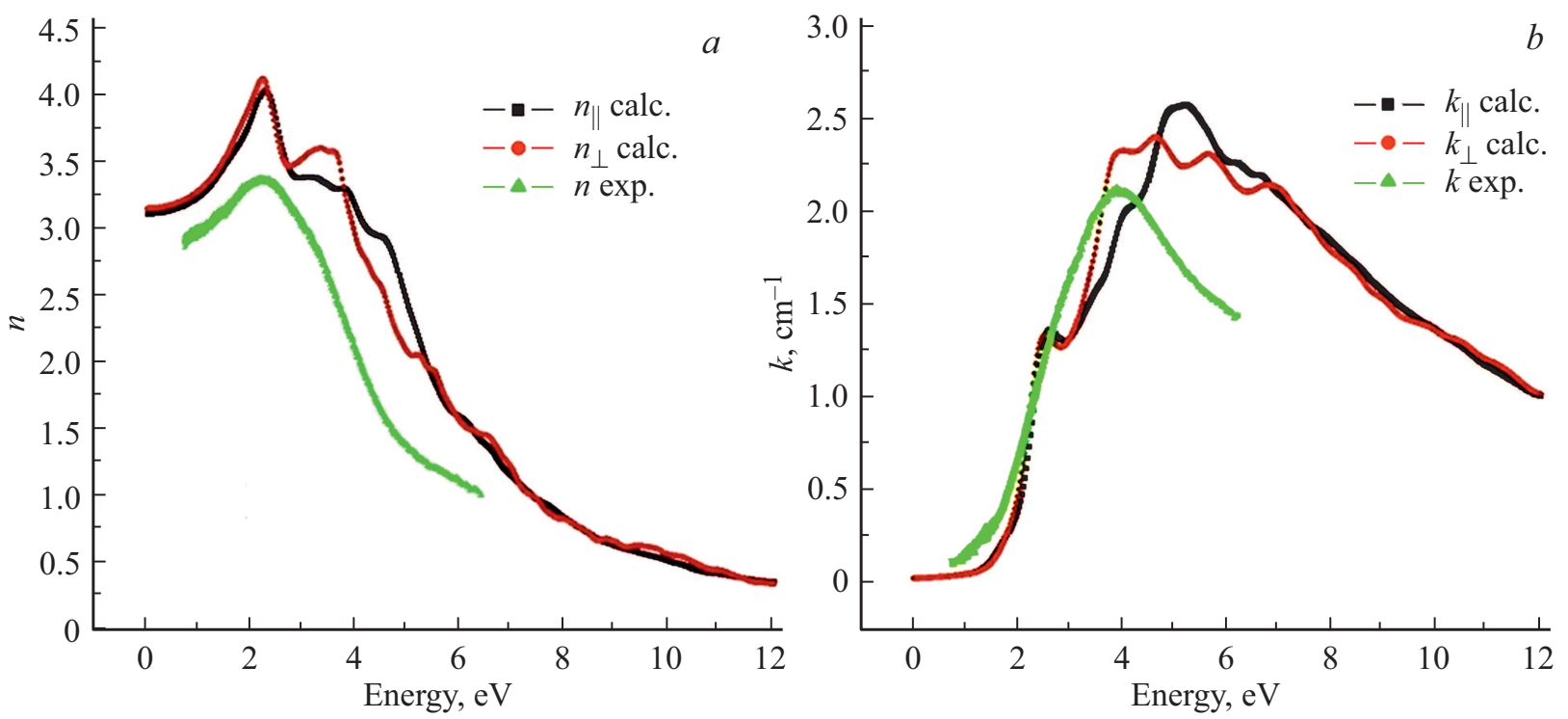

Рис. 6. Показатели преломления $n$ и экстинкции $k$ для $\mathrm{CdGa}_{2} \mathrm{Te}_{4}$.

Рассчитанные действительные и мнимые части диэлектрической функции $\mathrm{CdGa}_{2} \mathrm{Te}_{4}$ показаны на рис. 4. Основные пики рассчитанной действительной и мнимой части расположены около 4.0 и $2.0 \mathrm{eV}$, соответственно. На рис. 5 и 6 показаны измеренные и рассчитанные коэффициент поглощения $\alpha$, показатели преломления $n$ и экстинкции $k$, соответственно. Высокое значение коэффициента поглощения $\alpha$, его резкое увеличение в области энергий 1.5-3.15 eV свидетельствуют о прямом разрешенном характере запрещенной зоны. Из графика экспериментально определенного коэффициента поглощения $\alpha$ следует, что ширина запрещенной зоны составляет $\sim 1.6 \mathrm{eV}$ и $\mathrm{CdGa}_{2} \mathrm{Te}_{4}$ является прямозонным полупроводником. Это значение отлично согласуется с нашим теоретическим значением края поглощения, которое составляет около $1.5 \mathrm{eV}$, а также подтверждает наш теоретический результат о прямозонности данного кристалла и доказывает правильность рассчитанной зонной структуры. Кроме того, экспериментально определенный коэффициент поглощения $\alpha$ достигает плато на уровне около $5.0 \mathrm{eV}$. Эта особенность также хорошо воспроизводится нашим рассчитанным спектром на рис. 5 . Наконец, измеренный показатель преломления достигает максимума около $2.4 \mathrm{eV}$, что соответствует теоретическому положению пика, хотя теоретический пик намного шире, чем экспериментальный. Теоретическое и экспериментальное значения статического показателя преломления (значение показателя преломления при

Таблица 2. Некоторые диэлектрические параметры $\mathrm{CdGa}_{2} \mathrm{Te}_{4}$

\begin{tabular}{c|c|c|c|c}
\hline$\varepsilon_{1}(0)_{\perp}$ & $\varepsilon_{1}(0)_{\|}$ & $n(0)_{\perp}$ & $n(0)_{\|}$ & Литература \\
\hline 10.94 & 7.71 & 3.3 & 2.77 & {$[5]$} \\
9.53 & 9.56 & 3.09 & 3.09 & {$[2]$} \\
9.9 & 9.6 & 3.12 & 3.1 & Даннная работа (теория)
\end{tabular}

нулевой энергии) в случае $E_{\perp} c$ и $E_{\|} c$ составляют $\sim 3.0$, как показано на рис. 6. В табл. 2 приведены некоторые диэлектрические параметры $\mathrm{CdGa}_{2} \mathrm{Te}_{4}$. В целом все рассчитанные из первых принципов оптические свойства хорошо согласуются с экспериментальными данными.

\section{4. Колебательные свойства}

В примитивной ячейке $\mathrm{CdGa}_{2} \mathrm{Te}_{4}$ имеются 7 атомов и следовательно, фононный спектр имеет 21 нормальных фононных мод. Теоретико-групповой анализ дает следующие фононные моды: $\Gamma=3 A+6 B+6 E$, акустические моды $\Gamma_{\text {acoustic }}=B+E$ и оптические моды $\Gamma_{\text {optic }}=3 A+5 B+5 E$. Фононные моды симметрии $E$ двукратно вырождены.

Все оптические моды являются раман-активными, $B$ и $E$-моды активны также в инфракрасном отражении для $E_{\|} c$ и $E_{\perp} c$ поляризации соответственно. Моды $A$ являются неполярными рамановскими модами, в то время как моды $B$ и $E$ являются полярными модами и, следовательно, демонстрируют продольно-поперечное оптическое расщепление (LO-TO). Анализ вектора смещения атомов показывает, что в полносимметричных $A$ модах смещение атомов происходит, в основном, вдоль кристаллографических осей $x, y$ и $z$, а в модах $B$, атомы катионной решетки смещаются относительно анионной подрешетки вдоль тетрагональной оси $c$. Колебания мод симметрии $E_{x}$ и $E_{y}$ происходит преимущественно в плоскости $a b$, перпендикулярной оси $c$ вдоль кристаллографических осей $x$ и $y$ соответственно. На рис. 7, 8 приведены спектры комбинационного рассеяния и ИК-отражения, соответственно. Восемь из восемнадцати раман-активных мод и двенадцать ИК-активных мод обнаружены и идентифицированы (табл. 3). Результаты $a b$ initio расчетов фононного спектра были использованы 
Таблица 3. Экспериментально определенные и вычисленные с учетом макроскопического электрического поля в направлении [100] (в декартовых координатах) частоты оптических фононов в $\mathrm{CdGa}_{2} \mathrm{Te}_{4}$

\begin{tabular}{|c|c|c|c|c|c|c|c|c|c|c|}
\hline \multicolumn{4}{|c|}{$\mathrm{CdGa}_{2} \mathrm{~S}_{4}$} & \multicolumn{3}{|c|}{$\mathrm{CdGa}_{2} \mathrm{Se}_{4}$} & \multicolumn{4}{|c|}{$\mathrm{CdGa}_{2} \mathrm{Te}_{4}$} \\
\hline \multirow{2}{*}{ Mode } & \multirow{2}{*}{$\begin{array}{c}\omega_{\text {theo }}, \\
\mathrm{cm}^{-1}[10]\end{array}$} & \multicolumn{2}{|c|}{$\omega_{\exp }, \mathrm{cm}^{-1}$} & \multirow{2}{*}{$\begin{array}{c}\omega_{\text {theo }}, \\
\mathrm{cm}^{-1}[9]\end{array}$} & \multicolumn{2}{|c|}{$\omega_{\exp }, \mathrm{cm}^{-1}$} & \multirow{2}{*}{$\begin{array}{c}\omega_{\text {theo }}, \mathrm{cm}^{-1} \\
\text { данная } \\
\text { работа }\end{array}$} & \multicolumn{3}{|c|}{$\omega_{\exp }, \mathrm{cm}^{-1}$} \\
\hline & & IR [19] & $\mathrm{R}[10]$ & & IR [20] & $\mathrm{R}[21]$ & & $\begin{array}{c}\text { IR данная } \\
\text { работа }\end{array}$ & $\begin{array}{c}\mathrm{R} \text { данная } \\
\text { работа }\end{array}$ & IR [8] \\
\hline$A(\mathrm{R})$ & $\begin{array}{l}221.67 \\
311.54 \\
352.88\end{array}$ & & $\begin{array}{l}219 \\
310 \\
351\end{array}$ & $\begin{array}{l}138 \\
184 \\
199.8\end{array}$ & & $\begin{array}{l}140 \\
189.9 \\
266\end{array}$ & $\begin{array}{l}102 \\
132 \\
138.8\end{array}$ & & 132 & \\
\hline$B(\mathrm{IR}, \mathrm{R})$ & $\begin{array}{r}87.85 \\
165.66 \\
260.62 \\
316.54 \\
369.01\end{array}$ & $\begin{array}{l}162 / 168 \\
238 / 245 \\
262 / 269 \\
330 / 343 \\
367 / 393\end{array}$ & $\begin{array}{c}165 \\
- \\
260 \\
322 \\
392\end{array}$ & $\begin{array}{c}76.3 \\
123.49 \\
197.49 \\
210.41 \\
257.98\end{array}$ & $\begin{array}{l}122 \\
125 \\
198 \\
225 \\
275\end{array}$ & $\begin{array}{c}75 \\
125 / 127 \\
225 \\
247 \\
275\end{array}$ & $\begin{array}{r}63.4 \\
90.2 \\
161.2 \\
172.6 \\
228.1\end{array}$ & $\begin{array}{c}58 / 77 \\
98 \\
\\
182 \\
237\end{array}$ & $\begin{array}{r}57 \\
85 \\
114 \\
\\
234\end{array}$ & $\begin{array}{c}53 / 56 \\
93 / 98 \\
192 / 203 \\
217 / 228 \\
232 / 236\end{array}$ \\
\hline $\begin{array}{c}E_{x}(\mathrm{IR}, \mathrm{R}) \\
\mathrm{LO}\end{array}$ & $\begin{array}{r}88.75 \\
138.03 \\
253.48 \\
349.77 \\
377.64\end{array}$ & $\begin{array}{c}86 /- \\
136 / 144 \\
238 / 253 \\
318 / 346 \\
360 / 385\end{array}$ & $\begin{array}{c}83 \\
135 \\
243 \\
- \\
361\end{array}$ & $\begin{array}{l}68 \\
103.08 \\
184.35 \\
239 \\
266.45\end{array}$ & $\begin{array}{l}180 \\
242 \\
274\end{array}$ & $\begin{array}{c}67.6 \\
104.4 \\
181 \\
242 \\
278\end{array}$ & $\begin{array}{l}52.6 \\
75.8 \\
150.2 \\
197 \\
215.1\end{array}$ & $\begin{array}{r}78 \\
140 \\
201 \\
213\end{array}$ & $\begin{array}{l}162 \\
203\end{array}$ & $\begin{array}{l}142 \\
186 \\
205 \\
216 \\
247\end{array}$ \\
\hline $\begin{array}{c}E_{x}(\mathrm{IR}, \mathrm{R}) \\
\mathrm{TO}\end{array}$ & $\begin{array}{r}85.78 \\
137.33 \\
249.36 \\
321.92 \\
355.57\end{array}$ & & & $\begin{array}{r}66 \\
102 \\
179 \\
232 \\
246\end{array}$ & & & $\begin{array}{l}50.1 \\
75.8 \\
146.9 \\
192 \\
205.6\end{array}$ & $\begin{array}{l}155 \\
220\end{array}$ & & $\begin{array}{l}130 \\
169 \\
197 \\
210 \\
223\end{array}$ \\
\hline
\end{tabular}

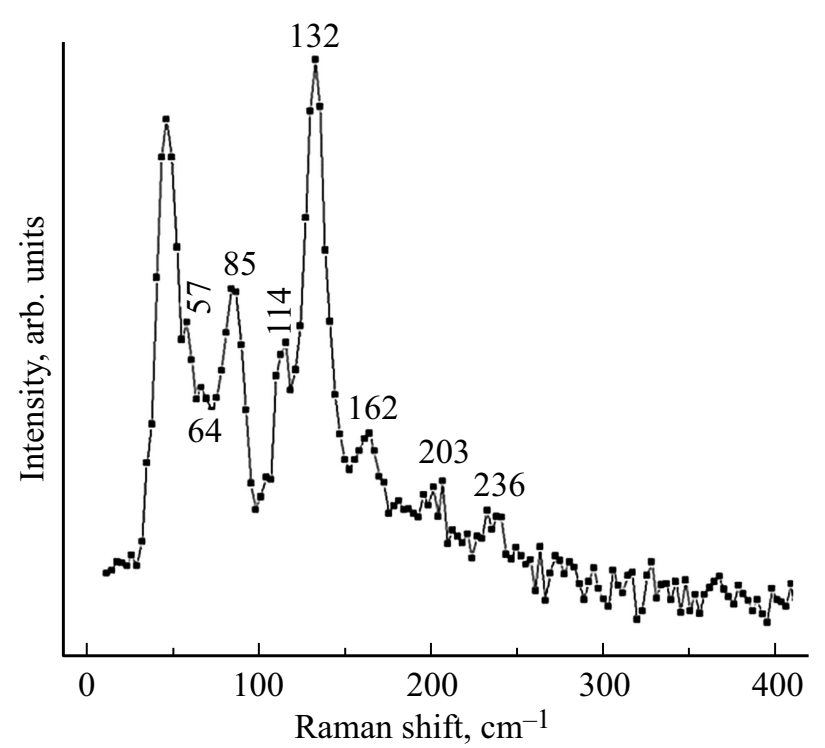

Рис. 7. Спектр комбинационного рассеяния света $\mathrm{CdGa}_{2} \mathrm{Te}_{4}$.

для детального определения и идентифицирования этих колебаний. Дисперсия фононных мод $\mathrm{CdGa}_{2} \mathrm{Te}_{4}$ вдоль высокосимметричных линий зоны Бриллюэна показана на рис. 9. Как видно из рис. 9, дисперсия оптических фононов во всех симметричных направлениях, кроме линии $P N$, является незначительной, что указывает на слабое межатомное взаимодействие особенно в направлении $\Gamma Z$ вдоль тетрагональной оси $c$. ИК-активные $B$ и $E$-моды вызывают LO-TO-расщепление в центре ЗБ и видны как разрывы в спектре (рис. 9).

Сравнение фононных спектров изоструктурных соединений $\mathrm{CdGa}_{2} \mathrm{Se}_{4}[9], \mathrm{CdGa}_{2} \mathrm{~S}_{4}[10]$ и $\mathrm{CdGa}_{2} \mathrm{Te}_{4}$, показывает, что характер дисперсии фононов у всех соединений в общем совпадают. Можно выделить два существенных различия: во-первых, с уменьшением атомной массы

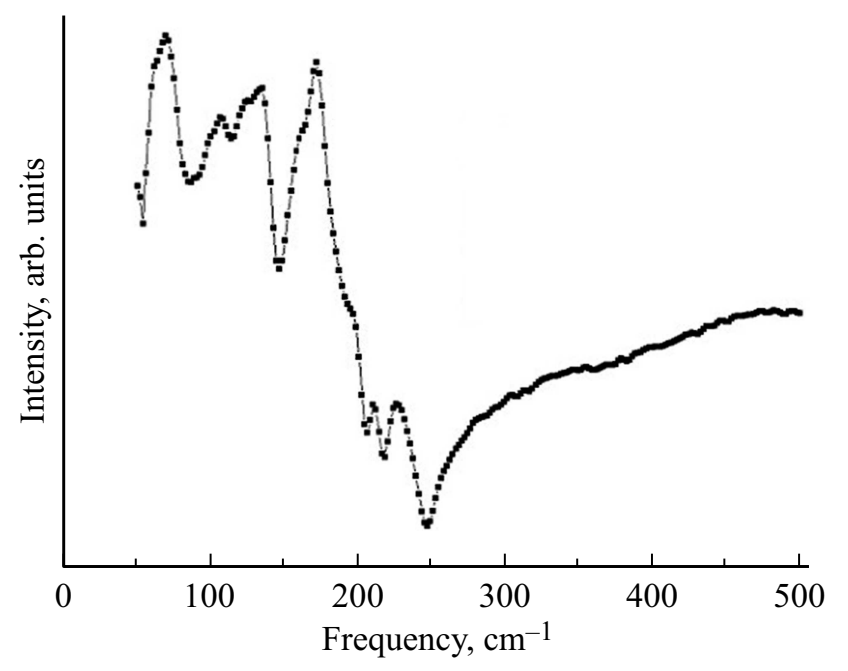

Рис. 8. Спектр ИК-отражения $\mathrm{CdGa}_{2} \mathrm{Te}_{4}$. 


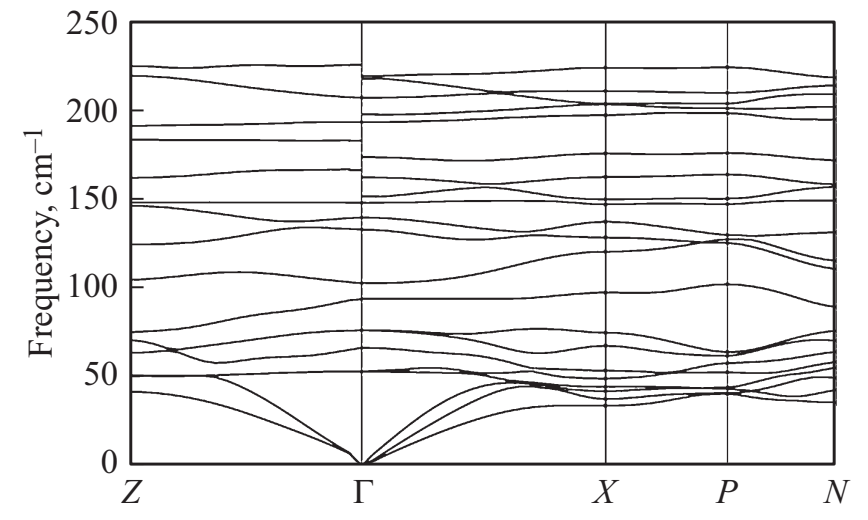

Pис. 9. Дисперсия фононов в $\mathrm{CdGa}_{2} \mathrm{Te}_{4}$.

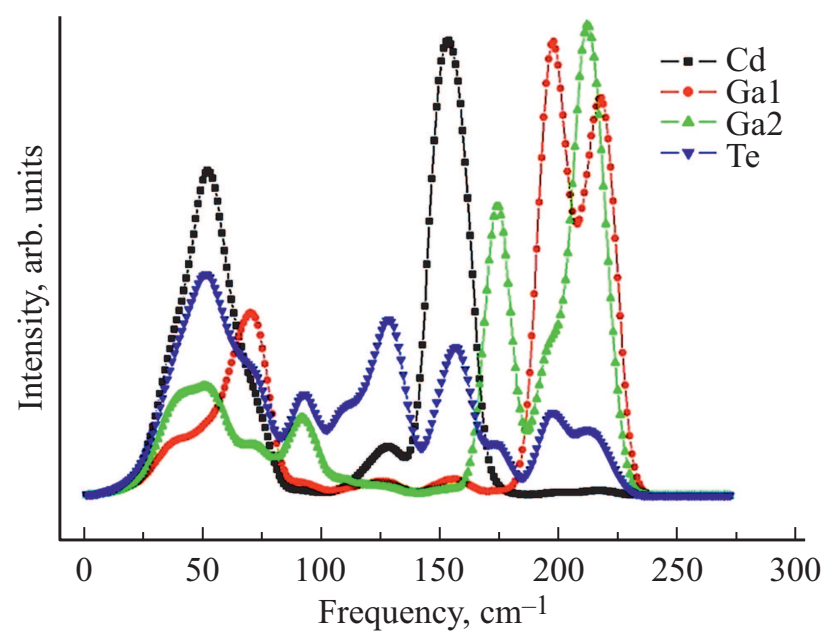

Рис. 10. Проектированные на атомы плотности фононных состояний в $\mathrm{CdGa}_{2} \mathrm{Te}_{4}$.

аниона значения частот соответствующих оптических мод уменьшаются, во-вторых, энергетические щели в фононном спектре $\mathrm{CdGa}_{2} \mathrm{~S}_{4}$, являющиеся результатом большой разности масс между атомами катиона $(\mathrm{Cd}, \mathrm{Ga})$ и аниона $(\mathrm{S})$, в фононном спектре $\mathrm{CdGa}_{2} \mathrm{Se}_{4}$ уменьшаются, а в фононном спектре $\mathrm{CdGa}_{2} \mathrm{Te}_{4}$ исчезают. А также, вклад халькогена в плотности фононных состояний сдвигается в сторону низких частот по мере увеличения атомной массы анионов ( $\mathrm{S}, \mathrm{Se}, \mathrm{Te})$. Проектированные на атомы плотности фононных состояний (Ph.PDOS) показаны на рис. 10. Анализ собственных векторов для каждого атома в примитивной ячейке и Ph.PDOS показывают, что акустические и низкочастотные оптические ветви с $B$ - и $E$-модами в частотном интервале от 0 до $60 \mathrm{~cm}^{-1}$ с максимумом при $50 \mathrm{~cm}^{-1}$ включают в себя движение атомов $\mathrm{Cd}$ и Те. Второй частотный интервал от 85 до $170 \mathrm{~cm}^{-1}$ с максимумом при $155 \mathrm{~cm}^{-1}$ в основном, связан с колебанием атома $\mathrm{Cd}$, с небольшим вкладом Те. В этом интервале атомы $\mathrm{Ga}$ в фононных колебаниях не участвуют.

Надо отметить, что атомы $\mathrm{Cd}$ участвуют в колебаниях только в частотных интервалах $20-85 \mathrm{~cm}^{-1}$ и
$140-170 \mathrm{~cm}^{-1}$. В области средних частот их вклад почти отсутствует. Высокочастотная третья область, в основном, связана с движением атомов $\mathrm{Ga}$ в двух кристаллографических позициях $(2 b)$ и $(2 c)$. Наиболее интенсивный пик спектра при $132 \mathrm{~cm}^{-1}$ соответствует $A$-моде в колебаниях которых участвуют только атомы аниона Те. Эта мода называется „дышащей“ поскольку она связана со симметричным колебанием анионов относительно стехиометрической вакансии. Низкочастотные и высокочастотные области, в основном, соответствуют модам $B$ и $E$, в то время как неполярные моды $A$ расположены в области средних частот. В табл. 3 приведены теоретически рассчитанные фононные частоты с учетом макроскопического электрического поля (с направлением [100] в декартовых координатах) и частоты фононов из экспериментальных исследований ИК- и КР-спектров $\mathrm{CdGa}_{2} \mathrm{Te}_{4}$. Как видно из таблицы, теоретически и экспериментально определенные частоты находятся в удовлетворительном согласии.

\section{5. Заключение}

Нами были проведены измерения комбинационного рассеяния и оптического поглощения в дефектном халькопирите $\mathrm{CdGa}_{2} \mathrm{Te}_{4}$, которые были сравнены с ab initio расчетами электронной структуры и динамики решетки. Наши ИК- и КР-измерения, дополненные теоретическими расчетами динамики решетки, позволили определить и идентифицировать наблюдаемые ИК- и КР-активные моды.

Результаты расчета и эксперимента показывают, что $\mathrm{CdGa}_{2} \mathrm{Te}_{4}$ является разрешенным прямозонным полупроводником с шириной запрещенной зоны $\sim 1.5 \mathrm{eV}$ и $\sim 1.6 \mathrm{eV}$ соответственно.

По мере увеличения атомной массы аниона $(\mathrm{S}, \mathrm{Se}, \mathrm{Te})$ в $\mathrm{CdGa}_{2} \mathrm{~S}_{4}, \mathrm{CdGa}_{2} \mathrm{Se}_{4}$ и $\mathrm{CdGa}_{2} \mathrm{Te}_{4}$ величины частот соответствующих оптических мод уменьшаются, энергетические щели в фононном спектре сужаются, а в $\mathrm{CdGa}_{2} \mathrm{Te}_{4}$ исчезают. Вклад колебаний атомов халькогена $(\mathrm{S}, \mathrm{Se}, \mathrm{Te})$ сдвигается в сторону низких частот по мере увеличения атомной массы атома халькогена. Низкочастотные и высокочастотные области в основном, соответствуют модам $B$ и $E$, в то время как неполярные моды $A$ расположены в области средних частот. Наиболее интенсивный пик спектра при $132 \mathrm{~cm}^{-1}$ соответствует А моде, которая связана со симметричным колебанием анионов относительно стехиометрической вакансии.

\section{Благодарности}

Авторы считают своим долгом выразить благодарность А.С. Бондякову (ОИЯИ, Россия) и Д.А. Кулиеву (Институт физики НАН Азербайджана), а также всему коллективу Дата-центра Института физики НАН Азербайджана, за предоставленные ресурсы и техническую поддержку теоретических расчетов. 


\section{Финансирование работы}

Работа выполнена при финансовой поддержке Фонда развития науки при Президенте Азербайджанской Республики (грант № EIF-BGM-3-BRFTF-2+/2017-15/02/1).

\section{Конфликт интересов}

Авторы заявляют, что у них нет конфликта интересов.

\section{Список литературы}

[1] А.Н. Георгобиани, С.И. Радауцан, И.М. Тигиняну. ФТП 19, 193 (1985).

[2] Jiao Zhao-Yong, Guo Yong-Liang. Chin. Phys. B 21, 12, 12310 (2012).

[3] Shunji Ozaki, Keiichi Muto, Sadao Adachi. J. Phys. Chem. Solids 64, 1935 (2003).

[4] Shunji Ozaki, Keiichi Muto, Hisatoshi Nagata, Sadao Adachi. J. Appl. Phys. 97, 043507 (2005).

[5] S. Mishra, B. Ganguli. Preprint submitted to Elsevier. (August 4, 2015).

[6] Mac Kinnon. In Tables of Numerical Data and Funtional Relationships in Science and Technology/ Ed O. Madelung. Landolt-Börnstein. New Serю, Group III, V. 17. SpringerVerlag, Berlin 124, (1985).

[7] X.S. Jiang, W.R. Lambrecht. Phys. Rev. B 69, 035201(2004).

[8] P.M. Nikolic, S.M. Stojilkovik. J. Phys. C 14, L551 (1981).

[9] 3.А. Джахангирли, Т.Г. Керимова, Н.А. Абдуллаев, И.А. Мамедова, Н.Т. Мамедов. ФТП 51, 5, 585 (2017).

[10] 3.А. Джахангирли, Т.Г. Керимова, И.А. Мамедова, Н.А. Абдуллаев, Н.Т. Мамедов. ФТТ 60, 11, 2265 (2018).

[11] H. Hahn, G. Frank, W. Kligler, A.D. Storger, Z. AnorgAllg. Chem. 279, 241 (1955).

[12] P. Gianozzi, S. de Gironcoli, P. Pavone, S. Baroni. Phys. Rev. B 43, 7231 (1991).

[13] S. Baroni, S. de Gironcoli, A. Dal Corso, P. Gianozzi. Rev. Mod. Phys. 73, 515 (2001).

[14] X. Gonze. Phys. Rev. B 55, 10337 (1997).

[15] X. Gonze, J.M. Beuken, R. Caracas, F. Detraux, M. Fuchs. G.M. Rignanese, L. Sindic, M. Verstraete, G. Zerah, F. Jallet. Comput. Mater. Sci. 25, 478 (2002).

[16] C. Hartwigsen, S. Goedecker, J. Hutter. Phys. Rev. B 58, 3641 (1998).

[17] J.P. Perdew, A. Zunger. Phys. Rev. B 23, 5048 (1981).

[18] H. Monkhorst, J. Pack. Phys. Rev. B 13, 5188 (1976).

[19] Т.Г. Керимова, Р.Х. Нани, Э.Ю. Салаев, В.Я. Штейншрайвер. ФТТ 21, 2791 (1979).

[20] Т.Г. Керимова, Р.Х. Нани, Э.Ю. Салаев, В.Я. Штейншрайвер. ФТП 21, 1961 (1979).

[21] Т.Г. Керимова, Р.Х. Нани, Н.Г. Дервишов, А.Ш. Хидиров, Ш.Н. Эфендиев. ФТТ 23, 638 (1981).

Редактор Д.В. Жуманов 